\title{
latrogenic Osteochondral Kissing Lesion After Transmalleolar Drilling of the Talar Dome
}

\section{A Case Report}

\section{Pieter D'Hooghe, MD* Kevin Deschamps, POD† Giovanni Matricali, MD‡}

\begin{abstract}
Ankle injuries can lead to early osteoarthrosis when diagnosis is delayed. The clinical presentation of an osteochondral lesion of the talar dome is often difficult to diagnose in the initial setup of an ankle sprain or fracture. Once the lesion is adequately imaged and staged, open or arthroscopic treatment can be initiated. We discuss the arthroscopic treatment of a posteromedial talar dome lesion in this case report. Transmalleolar drilling was performed to treat an osteochondral lesion of the talar dome in a 24-year-old female. Her ankle evolved toward a severe clinical and radiologic condition in which a combined talar and tibial (kissing) lesion was seen during her follow-up. Therefore, we recommend the use of retrograde drilling or arthroscopic microfracture with minimal iatrogenic risk, instead of transmalleolar drilling, as the treatment of choice in these specific osteochondral lesion of the talar dome cases. (J Am Podiatr Med Assoc 99(1): 54-57, 2009)
\end{abstract}

Ankle injuries are common and involve both osteocartilaginous and soft-tissue lesions. Patients with significant ankle injuries frequently fail to receive a detailed diagnosis because of normal radiologic findings, despite severe ligamentous tendon or osteocartilaginous lesions. The delay in diagnosis of an osteochondral lesion often leads to increased clinical complaints regarding pain and limited function. This condition can evolve toward early ankle osteoarthritis in up to $50 \%$ of the cases. ${ }^{1}$ Therefore, if symptoms persist, the clinician should have a high index of suspicion for an osteochondral lesion of the talar dome (OCLT). ${ }^{1-3}$

A variety of techniques have been used to treat these lesions over the years. Bone marrow-stimulating techniques have proven to be effective in many active patients with talar dome defects. Most authors have reported on the efficacy of arthroscopic drilling to improve clinical symptoms. ${ }^{4}$ Therefore, most treat-

\footnotetext{
*Orthopaedic Department, Stedelijk Ziekenhuis Roeselare, Roeselare, Belgium.

$\uparrow$ Division of Musculoskeletal Disorders, University Hospital of Leuven, Pellenberg, Belgium.

\$Orthopaedic Department, University Hospital of Leuven, Pellenberg, Belgium.

Corresponding author: Pieter D'Hooghe, MD, Orthopaedic Department, Stedelijk Ziekenhuis Roeselare, Brugsesteenweg 90, 8800 Roeselare, Belgium. (E-mail: pieter.orthopedie @gmail.com)
}

ment schedules prefer bone marrow-stimulating techniques (especially drilling) as the first choice surgical procedure. ${ }^{5,6}$ Despite recent innovations, certain areas, such as the posteromedial talar dome lesions, are still difficult to manage arthroscopically. The goal of the technique is to enter the defect with a Kirschner wire or drill in a perpendicular fashion. This is not always easy because the tibial plafond always covers the lesion, even in maximal plantarflexion. Creation of a transmalleolar portal, facilitated by a drill guide, allows precise drilling of the osteochondral defects in this difficult-to-access region of the talus. However, this technique inevitably creates iatrogenic lesions to intact cartilage on the tibial side of the malleolus, and theoretically it creates new cartilage lesions without sufficient healing capacity. 2, 5,7

To our knowledge, no cases concerning the presence of a kissing lesion after arthroscopically assisted transmalleolar drilling have been reported. We report on a patient that presented with this kind of lesion.

\section{Case Report}

A 24-year-old female patient suffered from a rightsided grade 1 open lateral malleolar fracture (Weber C) after a noncollision bicycle accident when she 
was 11 years old. She was initially treated with softtissue debridement and osteosynthesis of the fracture. After an initial adequate bony healing, she encountered persistent pain and swelling over her ankle during the rehabilitation period. Recently, she presented to the Orthopaedic Foot and Ankle Department, Stedelijk Ziekenhuis Roeselare, Roeselare, Belgium, with severe complaints of ankle pain, swelling, and function restraint toward plantar and dorsiflexion, especially during axial mechanical loads.

We obtained data showing that 1 year previously, the patient was treated with an arthroscopic-assisted transmalleolar drilling of a documented posteromedial talar dome osteochondral defect. Standard radiographs did not show any further bony abnormalities and a consolidation of the former fracture was observed (Fig. 1). Intraoperatively, there was no mention of an attempt to fill or treat the iatrogenic lesion, and the postoperative rehabilitation protocol included 6 weeks of partial weightbearing and passive motion physiotherapy.

Arthro-computed tomography (CT) showed a lack of healing tendency in the drilled talar dome tunnel and a new osteochondral lesion on the roof of the medial tibial recessus (Fig. 2). Furthermore, the arthroCT revealed articular cartilage damage over the entire anteromedial part of the tibial region along with two nearby subchondral defects (Fig. 3).
Therefore, besides the unsuccessful treatment of the talar cartilage lesion, the procedure also created a new kissing lesion onto the tibial cartilage with collateral damage to the subchondral bone near the defect.

\section{Discussion}

Various operative techniques for the treatment of OCLT have been reported and the results of each type of treatment have been relatively satisfactory. ${ }^{4,8} \mathrm{~A}$ study by Robinson et $\mathrm{al}^{9}$ showed that an OCLT often follows an ankle injury with articular damage and that it cannot be viewed in isolation because there will be other associated damage to the joint. The authors compared the transmalleolar drilling procedure with the excision and curettage procedure in OCLTs. Both techniques aim to revitalize the subchondral area beneath the defect, but they differ in approach. While the curettage procedure accesses the defect from the joint itself, the transmalleolar procedure drills a Kirschner wire into the defect from a transmalleolar direction. They state that the effect of both procedures is to debride the lesion and to allow bleeding from the base. Since this can be achieved with curettage alone, these authors did not see a need to violate the normal structure of the medial malleolus. An arthroscopic procedure, as compared to a malleolar osteotomy, shows many advantages because the
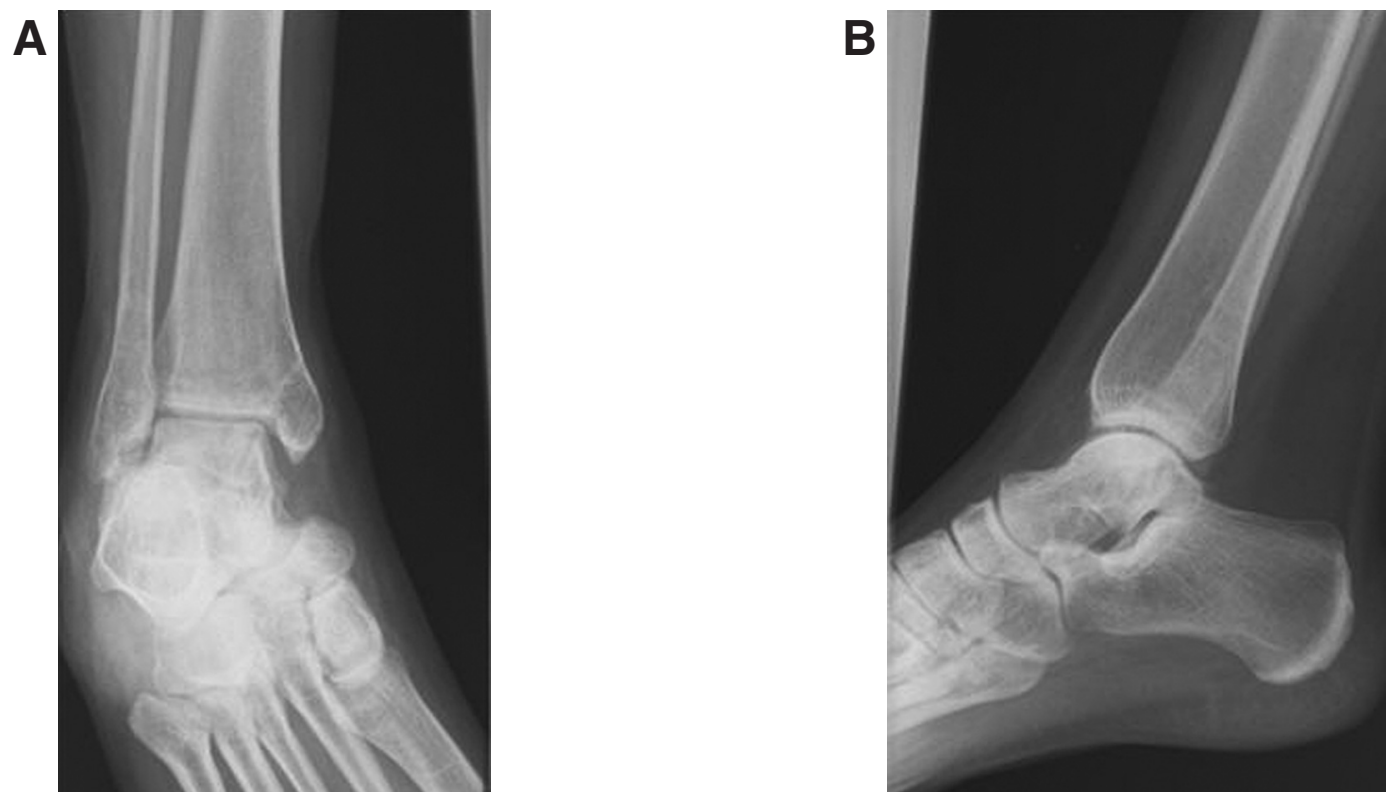

Figure 1. Postoperative anteroposterior radiograph (A) and sagittal radiograph (B) after arthroscopy-assisted transmalleolar drilling of an osteochondral lesion of the posteromedial talar dome. No further bony lesions can be detected and an adequate consolidation of the former fracture can be observed. Nevertheless, a discrete narrowing of the medial recessus can be noted on Figure $1 \mathrm{~A}$. 


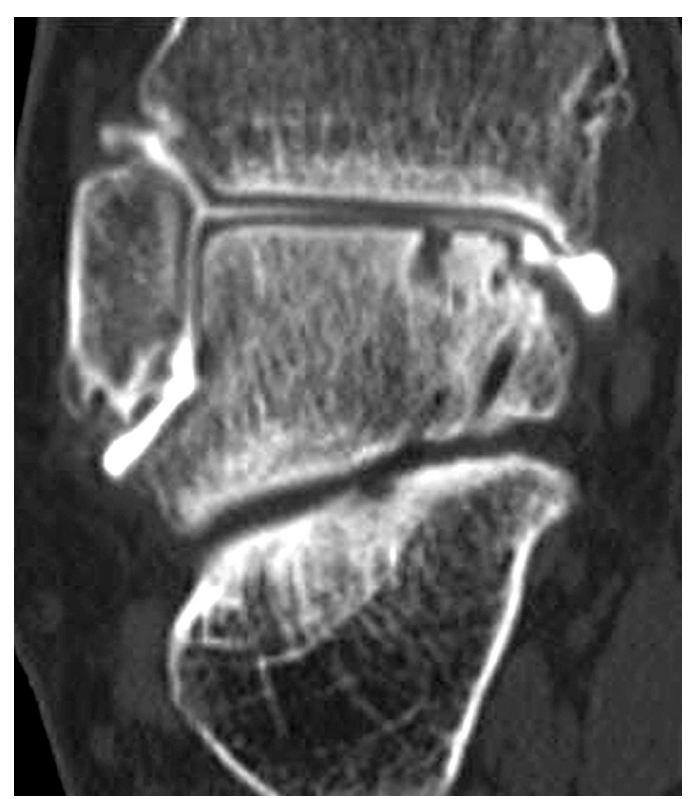

Figure 2. Arthro-computed tomography image showing a lack of healing tendency in the drilled talar dome tunnel and a new osteochondral lesion on the roof of the medial tibial recessus of the right ankle. It also shows two nearby subchondral defects in the talus and an irregular joint plane along the pin tracts in the posteromedial part of the talar dome.

procedure is less invasive, less painful, and allows early mobilization of the ankle joint. ${ }^{8}$

To reach the talar dome lesion, Angermann and Jensen ${ }^{10}$ propagated the transmalleolar drilling of the talar dome lesion, if the overlying talar articular cartilage appeared intact. However, a study by Kumai et $\mathrm{al}^{8}$ showed promising results when a retrograde talar drilling procedure was used. This technique shows a tendency toward healing in talar dome defects while the tibiotalar articular cartilage and surroundings are preserved. In their conclusion, the authors state that an early stable OCLT in a young patient should be considered an absolute indication for the retrograde drilling procedure. Another disadvantage of transmalleolar drilling is that the repaired cartilage is fibrous and will degenerate, eventually causing osteoarthritis. Unfortunately, long-term follow-up reports on arthroscopic drilling of an OCLT do not exist. ${ }^{11}$

Because of the anatomical considerations of the tibial plafond, transmalleolar drilling is theoretically a good alternative to curettage or excision, because it provides adequate access to the lesion with the proper drilling angle. Unfortunately, this technique may predispose the patient to iatrogenic kissing lesions with minimal healing capacity to the opposite tibial cartilage..$^{12}$ In the quest to avoid these complications,

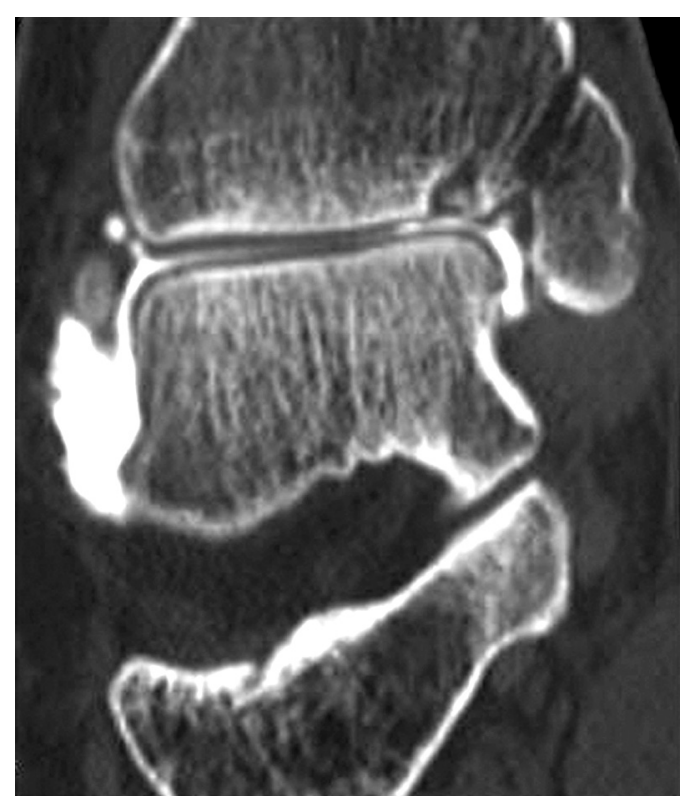

Figure 3. Arthro-computed tomography image showing the articular cartilage damage over the entire anteromedial part of the tibial roof over an extent of $0.9 \times$ $0.8 \mathrm{~cm}$. It shows tracks through the medial malleolus and a kissing lesion on the tibial roof at the intra-articular side of the track.

other techniques such as microfracturing and retrograde drilling (RD) seem very helpful.

Microfracture was first described by Steadman ${ }^{13}$ about 20 years ago. The technique creates multiple holes in the subchondral bone plate of the defect after proper debridement of its edges. This technique produces no heat, therefore possible thermal damage to the surrounding areas is virtually absent. Second, this procedure creates a rough surface on the subchondral bone that can allow the blood clot to adhere more easily. ${ }^{14}$ Third, the different angles of the awls available make it easier to create perpendicular holes in areas that are difficult to reach. ${ }^{12,14}$ We are not aware of any reports on complications that are specifically attributable to the technique of microfracture itself.

Retrograde drilling was first described by Lee and Mercurio in 1981, ${ }^{15}$ and Taranow et al ${ }^{16}$ proposed performing RD on an OCLT in 1999. The great advantage of $\mathrm{RD}$ is that it is possible to drill close to the subchondral bone without injuring the overlying articular cartilage. We are unaware of articles reporting (serious) complications due to the technique of drilling itself. Despite the recent enthusiasm for $\mathrm{RD}$, a recent study by Kono et al ${ }^{17}$ could not show a significant difference in the American Orthopaedic Foot and Ankle Society outcome score between the RD and the trans- 
malleolar approach 2 years postoperatively. The authors stated it might take a longer time to benefit from the RD advantages in the prevention of early osteoarthritis. ${ }^{17}$ Nevertheless, their study could significantly reveal the supremacy of RD in the arthroscopic assessment outcome score of their OCLTs. ${ }^{17,}{ }^{18}$ Considering the formerly outlined pros and cons of the various techniques, we prefer not to violate healthy cartilage, and we aim to use a microfracturing technique or retrograde drilling in OCLTs.

\section{Conclusions}

We describe a kissing lesion after transmalleolar drilling for OCLT in the ankle joint of a 24-year-old female. This causes severe pain, intra-articular swelling, and clinical disability together with a therapeutic dilemma. Therefore, this case illustrates some of the advantages of the microfracture and RD techniques as compared to the transmalleolar technique for OCLT. Microfracture and RD techniques both show effective resolution of these lesions without placing the patient at greater risk for iatrogenic lesions associated with transmalleolar drilling.

\section{Financial Disclosure: None reported.} Conflict of Interest: None reported.

\section{References}

1. Nelson SC, HaYcock DM: Arthroscopy-assisted retrograde drilling of osteochondral lesions of the talar dome. JAPMA 95: 91, 2005.

2. Barnes CJ, Ferkel RD: Arthroscopic debridement and drilling of osteochondral lesions of the talus. Foot Ankle Clin 8: 243, 2003.

3. SCHEnck RC, Goodnight JM: Osteochondritis dissecans. J Bone Joint Surg Am 78: 439, 1996.

4. Verhagen RA, Struijs PA, Bossuyt PM, et Al: Systematic review of treatment strategies for osteochondral defects of the talar dome. Foot Ankle Clin 8: 233, 2003.

5. Zengerink M, Szerb I, Hangody L, Et AL: Treatment of osteochondral ankle defects. Foot Ankle Clin 11: 331, 2006.

6. Giannini S, VANnini F: Operative treatment of osteochondral lesions of the talar dome: current concept review. Foot Ankle Int 25: 168, 2004.

7. Stone JW: Osteochondral lesions of the talar dome. J Am Acad Orthop Surg 4: 63,1996.

8. Kumai T, Takakura Y, Higashiyama I, et al: Arthroscopic drilling for the treatment of osteochondral lesions of the talus. J Bone Joint Surg 81: 1229, 1999.

9. Robinson DE, Winson IG, Harries WJ, ET aL: Arthroscopic treatment of osteochondral lesions of the talus. J Bone Joint Surg 85: 989, 2003.

10. Angermann P, Jensen P: Osteochondritis dissecans of the talus: long-term results of surgical treatment. Foot Ankle 10: 161, 1989.

11. TAkaO M, Ochi M, Naito K, ET al: Arthroscopic drilling for chondral, subchondral, and combined chondral-subchondral lesions of the talar dome. Arthroscopy 19: 524, 2003.

12. Giannini S, Buda R, FAldini C, ET AL: Surgical treatment of osteochondral lesions of the talus in young active patients. J Bone Joint Surg 87: 28, 2005.

13. Steadman JR, Rodkey WG, Rodrigo JJ: Microfracture: surgical technique and rehabilitation to treat chondral defects. Clin Orthop Relat Res 391 (suppl): S362, 2001.

14. Hangody L, Kish G, Karpati Z, Et al: Treatment of osteochondritis dissecans of the talus: use of the mosaicplasty technique-a preliminary report. Foot Ankle Int 18: $628,1997$.

15. Lee CK, Mercurio C: Operative treatment of osteochondritis dissecans in situ by retrograde drilling and cancellous bone graft: a preliminary report. Clin Orthop Relat Res 158: 129, 1981.

16. Taranow WS, Bisignani GA, Towers JD, Et aL: Retrograde drilling of osteochondral lesions of the medial talar dome. Foot Ankle Int 20: 474, 1999.

17. Kono M, TAKaO M, NaIto K, ET AL: Retrograde drilling for osteochondral lesions of the talar dome. Am J Sports Med 34: 1450, 2006.

18. Hunt SA, Sherman O: Arthroscopic treatment of osteochondral lesions of the talus with correlation of outcome scoring systems. Arthroscopy 19: 360, 2003. 